\title{
Clues from 4U 0142+61 on supernova fallback disc formation and precession
}

\author{
Catia Grimani, $1,2 \star$ \\ ${ }^{1}$ DiSPeA, University of Urbino Carlo Bo, Urbino (PU), Italy \\ ${ }^{2}$ Italian Institute for Nuclear Physics, Florence, Italy
}

Accepted XXX. Received YYY; in original form ZZZ

\begin{abstract}
The NuSTAR experiment detected a hard X-ray emission (10-70 keV) with a period of $8.68917 \mathrm{~s}$ and a pulse-phase modulation at $55 \mathrm{ks}$, or half this value, from the anomalous X-ray pulsar (AXP) 4U 0142+61. It is shown here that this evidence is naturally explained by the precession of a Keplerian supernova fallback disc surrounding this AXP. It is also found that the precession of discs formed around young neutron stars at distances larger than those considered in the past, may constitute almost neglected sources of gravitational waves with frequencies belonging to the sensitivity bands of the future space interferometers LISA, ALIA, DECIGO and BBO. In this work the gravitational wave emission from precessing fallback discs possibly formed around young pulsars such as Crab in a region extending beyond $8 \times 10^{7} \mathrm{~m}$ from the pulsar surface is estimated. It is also evaluated the role that infrared radiation emission from circumpulsar discs may play in contributing to Inverse Compton Scattering of TeV energy positrons and electrons. Extensive observational campaigns of disc formation around young and middle aged pulsars may also contribute to solve the long-standing problem of a pulsar origin for the excess of positrons in cosmic rays observed near Earth above $7 \mathrm{GeV}$. In the near future the James Webb telescope, with unprecedented near and mid-infrared observation capabilities, may provide direct evidence of a large sample of supernova fallback discs.
\end{abstract}

Key words: gravitational waves - protoplanetary discs - cosmic rays - pulsars:general

\section{INTRODUCTION}

Monte Carlo simulations indicate that supernova fallback discs may likely form near the light cylinder (LC) of pulsars with birthperiods > than $40 \mathrm{~ms}$ (Jiang \& Li 2005). Observational clues reported in Grimani (2016) indicate that this is the case for the majority of young pulsars. However, depending on the angular momentum of fallback matter, discs may also form at different distances from the pulsar (Currie \& Hansen 2007). The role of disc formation in affecting the evolution of pulsars (Benli \& Ertan 2017) and magnetars was extensively discussed in the literature to explain observations from soft gamma repeaters (SGRs, Tong et al. 2011), anomalous Xray pulsars (AXPs, Chatterjee et al. 2000; Alpar 2001; Ertan et al. 2009), rotating radio transients (RRATs, Li 2006; Gençali \& Ertan 2020), X-ray dim isolated neutron star (XDINS, Ertan et al. 2014; Özcan et al. 2020) and central compact objects (CCOs, Erdeve et al. 2009; Benli \& Ertan 2018).

The presence of a disc was claimed around the AXP 4U 0142+61 on the basis of the Spitzer satellite observations and earlier data (Wang et al. 2006; Ertan et al. 2007). While this discovery was questioned in the literature (Durant \& van Kerkwijk 2006), the NuSTAR experiment detected an X-ray emission modulated at $55 \mathrm{ks}$, or half this value, from the same AXP (Makishima et al. 2018). It is shown here that this modulation is compatible with the presence of a Keple-

^ E-mail: catia.grimani@uniurb.it rian precessing disc with the characteristics observed by Wang et al. (2006) hiding periodically the emission region. The possible formation of a disc around the magnetar 1E 2259+586 was also reported in Kaplan et al. (2009). A hundreds of AU region emitting infrared radiation around the X-ray thermal isolated neutron stars (XTINS) RX J0806.4-4123 was detected with the Hubble telescope (Posselt et al. 2018). This observation is compatible with the presence of a huge disc surrounding the pulsar.

The presence of a disc around the RRAT B0656+14 was reported in Perna et al. (2000). In Grimani (2013) it was pointed out that a precessing disc could have explained the transient emission from this pulsar.

In the past, however, several campaigns of observations did not allow to detect a large sample of circumpulsar discs (Wolszczan 2008), possibly because of low instrument sensitivities and disc inclinations with respect to detectors field of view.

The TeV pulsed photon emission observed with the MAGIC experiment (Ansoldi et al. 2016) in a region extending beyond $8 \times 10^{7}$ $\mathrm{m}$ from the Crab pulsar is consistent with $\mathrm{TeV} \mathrm{e}^{+}$and $\mathrm{e}^{-}$scattering infrared photons to $\mathrm{TeV}$ energies. In the case of disc formation in that region both pulsar and disc infrared radiation may contribute to this process. The hypothesis of a disc surrounding Crab just beyond the LC was discussed in Menou et al. (2001).

A step forward in the knowledge of pulsar and magnetar environments may also provide precious insights on positron observations in cosmic rays gathered near Earth showing an increasing excess of 
these particles with respect to the estimated secondary component above $7 \mathrm{GeV}$, with a drop-off just below $300 \mathrm{GeV}$ and a possible cutoff at about $800 \mathrm{GeV}$ (Aguilar et al. 2019; Diesing \& Caprioli 2020; Huang et al. 2020). While an astrophysical or an exotic origin of the excess of positrons in cosmic rays remains to be settled (see for instance Harding \& Ramaty (1987); Zhang \& Cheng (2001); Grimani (2004, 2007); Feng \& Zhang (2018); Manconi et al. (2020)), observations are compatible with sources of $\mathrm{TeV} \mathrm{e}^{-}-\mathrm{e}^{+}$located between 100 and 800 pc from the Solar System (Attallah 2016).

The James Webb telescope (JWT, Gardner et al. 2006), scheduled to launch in the fall 2021, will definitely open the infrared observational window on disc formation around magnetars and pulsars.

Following the NuSTAR observations, this paper aims to evaluate the consequences of Keplerian discs precessing around pulsars and magnetars in generating gravitational waves with frequencies belonging to the sensitivity bands of the future LISA (Amaro-Seoane et al. 2017), ALIA (Ni 2016), BBO (Cutler \& Harms 2006), and DECIGO (Kawamura et al. 2020) space interferometer sensitivity bands.

In Grimani (2016) the gravitational wave emission from precessing discs formed near the LC of young and middle aged pulsars was considered and discussed. In the same paper it was also shown that gravitational waves emitted by these precessing discs could have been detected with the second generation LISA-like space interferometers such as BBO and DECIGO, presenting maximum sensitivities near $1 \mathrm{~Hz}$. The NuSTAR observations are consistent with the scenario of disc formation and precession proposed in Grimani (2016) but may also suggest that disc formation occur at larger distances than previously considered. DECIGO remains the favored interferometer to detect gravitational waves generated by precessing discs formed around pulsars possibly up to distances exceeding tens of $\mathrm{kpc}$ from Earth. In particular, depending on the actual dimensions and masses of discs and precession angles, observations carried out with the future space interferometers may help in composing the puzzle of disc role in affecting the environment of neutron stars (NSs) especially in respect to high-energy electrons and positrons undergoing inverse Compton (IC) scattering of soft photons before escaping the source region (Grimani 2013, 2016).

In Section 2 characteristics of the disc observed around $4 \mathrm{U}$ $0142+61$ and speculations about a disc possibly formed around Crab are reported. In Section 3 the precession of a disc around 4U 0142+61 is considered to explain the modulated X-ray emission observed from this magnetar. Alternative scenarios involving pulsar free and forced precession have been also considered. In Section 4 gravitational wave emission from precessing discs around pulsars and magnetars is reestimated following the observations of the NuSTAR experiment. Finally, in Section 5 the infrared emission from a disc formed around a young pulsar such as Crab is compared to that of the host neutron stars in the disc region in order to estimate if $\mathrm{TeV}$ electron and positron propagation could be affected by the disc presence.

\section{FALLBACK DISC CHARACTERISTICS}

\section{$2.14 \mathrm{U} 0142+61$}

The disc surrounding the AXP 4U 0142+61 observed by Wang, Chakrabarty and Kaplan Wang et al. (2006) has a mass of $5.97 \times 10^{25}$ $\mathrm{kg}$ and presents inner $\left(R_{I}\right)$ and outer $\left(R_{O}\right)$ radii of $2.02 \times 10^{9} \mathrm{~m}$ and $6.75 \times 10^{9} \mathrm{~m}$, respectively. The disc height (h) is set here equal to $0.035 \times R_{I}$ according to Hawley \& Krolik (2018). The observed disc temperature $\left(T_{D}\right)$ is $920 \mathrm{~K}$. The associated blackbody radiation according to Wien's law, $\lambda_{\max } \mathrm{T}=2.898 \times 10^{-3} \mathrm{~m} \mathrm{~K}$ with $\lambda_{\max }=3.15 \times 10^{-6} \mathrm{~m}$, peaks in the mid-infrared range. The AXP $4 \mathrm{U} 0142+61$ has a surface temperature of $0.309 \pm 0.001 \mathrm{keV}$ equivalent to a blackbody temperature $\left(T_{N S}\right)$ of $3.59 \times 10^{6} \mathrm{~K}$ (Güver et al. 2008). By using the Stefan-Boltzmann law, $\sigma \mathrm{T}^{4}=5.67 \times 10^{-8} \mathrm{~T}^{4} \mathrm{~W}$ $\mathrm{m}^{-2}$, and by considering $10 \mathrm{~km}$ for the magnetar radius $\left(R_{N S}\right)$ the temperature of the disc $\left(T_{D}\right)$ illuminated by the pulsar can be inferred from:

$\frac{R_{N S}^{2}}{R_{I}^{2}} \sigma T_{N S}^{4} A_{D}=A_{D 1} \sigma T_{D}^{4}$,

where $A_{D}$ and $A_{D 1}$ are the area of the disc illuminated by the pulsar and the area of the disc emitting as a blackbody, respectively. Several uncertainties limit the precision of this estimate: a) the actual radius of pulsars/magnetars ranging between 10 and $14 \mathrm{~km}$ for a typical 1.4 solar mass star (Steiner et al. 2018), b) the exact pulsar/magnetar blackbody radiation at the equatorial region (where the disc plausibly forms) being about $30 \%$ smaller than that of the hot spots and polar cap (Aguilera et al. 2008) and c) the actual thickness and energy absorbed by the disc (Benli et al. 2015; Shakura \& Sunyaev 1973; Lu \& Cheng 2002). Finally, the NS blackbody photon gravitational redshift would be of the order of $10 \%$. Even by taking into account these considerations, the conclusion about the energy of photons reaching the disc would not change sensibly. The detected blackbody temperature of the disc would result of $920 \mathrm{~K}$ from equation (1) if, as plausible, the energy absorbed by the disc surrounding the magnetar is $5 \%$ of the illuminating energy.

\subsection{Crab}

The MAGIC experiment observed a pulsed gamma-ray emission from the Crab pulsar up to $1.5 \mathrm{TeV}$ energy (Ansoldi et al. 2016). The gamma-ray emission pulse profile presents two peaks and the energy differential fluxes show spectral indices close to 3 . These observations are consistent with electrons and positrons with Lorentz factors $\gamma>5 \times 10^{6}$ undergoing IC scattering with low-energy photons, typically infrared radiation, mainly concentrated at distances well beyond $50 \mathrm{LC}$ radii from the Crab surface. The process that accelerates electrons and positrons is not well understood (Ansoldi et al. 2016), but the infrared emission from a disc, in addition to pulsar magnetospheric and/or pulsed infrared radiation, may play a role in affecting the high-energy electron and positron propagation. We are aware that this is only a possible scenario but it is proposed by considering that the formation of a disc around Crab should be favoured well beyond the typical LC distance as proposed by Menou et al. (2001) because the temperature falls below $2000 \mathrm{~K}$, similarly to the case of $4 \mathrm{U} 0142+61$.

The upper limit of the blackbody temperature of the Crab pulsar is 2.1 MK (Tennant et al. 2001). According to the Wien's law, the blackbody radiation peaks in the X-ray band at $143 \mathrm{eV}$ and the average energy is:

$\epsilon_{a v g}=\frac{\sigma c^{2} h^{3} T}{2.4052 \pi k^{3}}=490 \mathrm{eV}$.

As recalled above, the gravitational redshift would not affect significantly the described scenario. By using the same considerations applied to $4 \mathrm{U} 0142+61$ in the previous Section, if a disc surrounds Crab beyond 50 LC distance from the pulsar surface, the disc inner radius would be $>8 \times 10^{7} \mathrm{~m}$ and the thickness could be set to $2.8 \times 10^{6}$ $\mathrm{m}$ as a lower limit. Given the blackbody temperature of Crab and a disc absorbing $10^{-3}$ of the illuminating X-ray energy, the nominal 


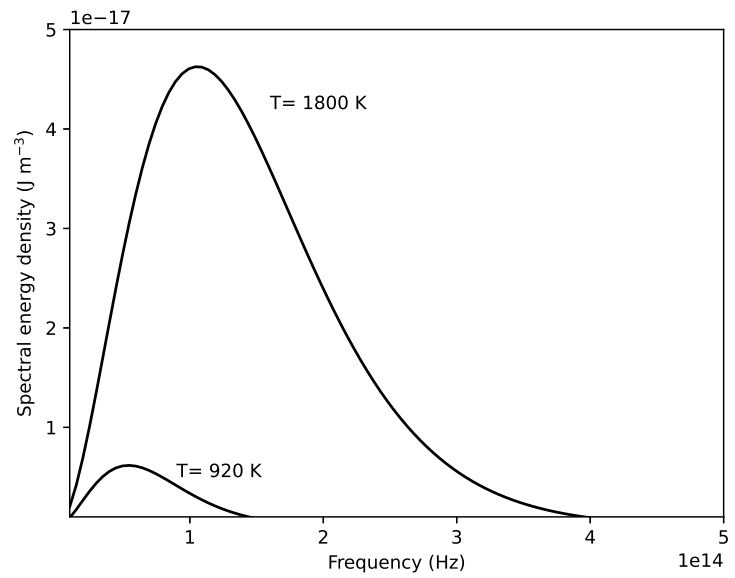

Figure 1. Blackbody radiation spectral energy density for disc temperature of $920 \mathrm{~K}$ and $1800 \mathrm{~K}$.

inner temperature of the disc would be about $1800 \mathrm{~K}$ according to equation 1 . The blackbody radiation of discs of $1800 \mathrm{~K}$ and $920 \mathrm{~K}$ is reported in Figure 1 for comparison with Figure 3 in Wang et al. (2006).

An absorption of the illuminating energy of $10^{-2}$ as indicated by the blackbody radiation of the disc surrounding $4 \mathrm{U} 0142+61$ would lead to a similar temperature of the disc in case the same would be thicker or larger than assumed. Moreover, the disc may extend well beyond $50 \mathrm{LC}$ where the temperature has values similar or smaller than that of the disc observed around $4 \mathrm{U} 0142+61$. The comparison between disc and pulsar infrared emission in the disc region is discussed in Section 5.

\section{IMPLICATIONS OF A KEPLEARIAN PRECESSING DISC SURROUNDING 4U 0142+61}

The modulated X-ray emission at 55(27.5) ks observed with the NuSTAR experiment from $4 \mathrm{U} 0142+61$ was explained in Makishima et al. (2018) with a magnetar free precession induced by the star magnetic field of about $10^{16} \mathrm{G}$. Nevertheless, this magnetic field value exceeds by far previous estimates reported in the literature of $(4.75 \pm 0.02) \times 10^{14} \mathrm{G}$ (Güver et al. 2008). Forced precession of a pulsar due to the action of a surrounding accretion or fallback disc was proposed by Tong et al. (2020). If this process explains the NuSTAR observations from $4 \mathrm{U} 0142+61$, the angular momentum of the disc must be $>$ than that of the pulsar and the precession period is expected to appear consistent with the observations. By defining $I_{4 U} 0142+61$ and $I_{D}$ the moments of inertia of the magnetar and of the disc, respectively, as follows:

$$
I_{4 U 0142+61}=\frac{2}{5} M_{4 U} 0142+61 R_{4 U}^{2} 0142+61=1.12 \times 10^{38} \mathrm{~kg} \mathrm{~m}^{2}
$$

$I_{D}=\frac{1}{2} M_{D}\left(R_{I}^{2}+R_{O}^{2}\right)=1.48 \times 10^{45} \mathrm{~kg} \mathrm{~m}^{2}$,

where $R_{4 U} 0142+61=R_{N S}$ and $M_{4 U} 0142+61=2.8 \times 10^{30} \mathrm{~kg}$, then the angular momenta of the magnetar $\left(L_{4 U 0142+61}\right)$ and of the disc $\left(L_{D}\right)$ can be calculated as indicated below:

$L_{4 U 0142+61}=I_{4 U 0142+61} \omega_{4 U 0142+61}$,

$L_{D}=I_{D} \omega_{D}$

The angular frequency of the magnetar is $\omega_{4 U 0142+61}=0.723 \mathrm{~Hz}$ and the Keplerian angular frequency of the disc, $\Omega_{K}=\omega_{D}$ is determined from the equation $V=\Omega_{K} R_{I}$, where $V=\sqrt{\left(G M_{4 U 0142+61}\right) / R_{I}}$ is the disc velocity at the inner radius, $R_{I}$, and $G$ is the universal gravitational constant equal to $6.67 \times 10^{-11} \mathrm{~N} \mathrm{~m}^{2} \mathrm{~kg}^{-2}$. As recalled in the previous Section, the inner radius of the disc around $4 \mathrm{U} 0142+61$ is $2.02 \times 10^{9} \mathrm{~m}$, therefore $V$ is $3.0 \times 10^{5} \mathrm{~m} \mathrm{~s}^{-1}$ and $\Omega_{K}=$ $\sqrt{\left(G M_{4 U 0142+61}\right) / R_{I}^{3}}=1.505 \times 10^{-4} \mathrm{~s}^{-1}$. As a result,

$L_{4 U} 0142+61=8.10 \times 10^{37} \mathrm{~kg} \mathrm{~m}^{2} \mathrm{~s}^{-1}$

and

$L_{D}=2.23 \times 10^{41} \mathrm{~kg} \mathrm{~m}^{2} \mathrm{~s}^{-1}$.

The disc may induce the magnetar under precession, however the precession period, estimated according to Qiao et al. (2003) as:

$P=\frac{2 \pi}{|\dot{\phi}|}$

where

$|\dot{\phi}|=\frac{45 M_{D} \cos \theta^{\prime} \omega_{4 U 0142+61}}{32 \pi \rho R_{I}^{3}}$,

is $2.77 \times 10^{20} \mathrm{~s}$, by reasonably assuming in the above equation $\theta^{\prime}=15^{\circ}$ and $\rho=10^{17} \mathrm{~kg} \mathrm{~m}^{-3}$ for the neutron star density. It is recalled here that $\theta^{\prime}$ represents the angle formed by the rotation axis of the pulsar and the perpendicular to the disc plane. The result obtained is definitely not in agreement with the NuSTAR observations. The precession period increases with $\theta^{\prime} \rightarrow \pi / 2$ or if the disc radius increases. The model by Tong et al. (2020) applies better to scenarios involving heavy discs formed near young pulsars.

In the paper by Makishima et al. (2018) our previous work (Grimani 2016) was mentioned to point out that our results were not in agreement with the NuSTAR observations. However, this is not the case. In the following it is shown that the free precession of a Keplerian disc (due to a misalignment of the symmetry and the angular momentum axes) with the characteristics reported in Section 2 would naturally explain the NuSTAR observations. If $I_{1}, I_{2}$ and $I_{3}$ are the principal moments of inertia of the disc with respect to the principal axes, $x_{1}, x_{2}$ and $x_{3}$ fixed in the disc and $\Omega_{3}$ is the angular velocity along the symmetry axis $x_{3}$, then $\Omega_{3}$ is commonly assumed equal to the Keplerian frequency $\Omega_{K}$ estimated at the inner disc radius, $R_{I}$.

The precession pulsation, $\omega_{P}$, is determined as follows:

$\omega_{P}=\frac{I_{3}}{I_{1} \cos \theta} \Omega_{K}$,

where

$I_{1}=I_{2}=\frac{1}{2} I_{3}=\Delta I$ 
and $I_{3}=I_{D}$. If $\theta$ is the wobble angle defined as the angle between the angular momentum and the symmetry axis of the disc, it is found that for $\theta \simeq 0, \cos \theta \simeq 1$ and $\omega_{P} \simeq 2 \Omega_{K}=3.01 \times 10^{-4} \mathrm{~Hz}$.

Therefore, the precession period of the disc detected by Spitzer would be:

$T_{P}=\frac{2 \pi}{\omega_{P}}=20.9 \mathrm{ks}$,

in a fair agreement with half period $(27.5 \mathrm{ks})$ of the X-ray modulation observed by NuSTAR from 4 U $0142+61$.

\section{GRAVITATIONAL WAVE EMISSION FROM PRECESSING DISCS}

In Grimani (2016) and references therein it was shown that the frequencies of gravitational waves generated by precessing circumpulsar discs are $\omega_{G W}$ and $2 \omega_{G W}$, where $\omega_{G W}$ is defined as follows:

$\omega_{G W}=\omega_{P}$.

Under the small wobble angle approximation $\left(\theta=10^{\circ}\right)$, the gravitational wave frequencies associated with a Keplerian disc precessing around the magnetar $4 \mathrm{U} 0142+61$ would be $3.1 \times 10^{-4} \mathrm{~Hz}$ and $6.2 \times 10^{-4} \mathrm{~Hz}$ that, as it can be observed in Figure 2, lie in the LISA sensitivity band, nominally ranging between $10^{-1}$ and $10^{-4}$ Hz. It is worthwhile to point out that, due to the LISA Pathfinder (Armano et al. 2018) encouraging results, the frequency range of the LISA observations may be likely extended down to $10^{-5} \mathrm{~Hz}$.

The gravitational wave amplitudes $(h)$ generated by precessing discs depend on the inclination angle $(i)$ of the disc angular momentum with respect to the line of sight (see Grimani (2016) and references therein for details). For instance, in the case $i \simeq 0$ and $\theta$ small (Lee et al. 2004):

$h \simeq \frac{G}{c^{4}} \frac{\omega^{2}}{r} \Delta I \theta^{2}$,

where $r$ is the distance between the precessing disc and the observer and $\Delta I$ is defined in equation 12 .

The maximum distance at which the future interferometers for gravitational wave detection in space will observe precessing discs depends on the principal moments of inertia which, in turn, depend on the disc masses and dimensions. Mass discs up to $10^{29} \mathrm{~kg}$ are considered here according to Qiao et al. (2003) and references therein. The amplitudes of the gravitational waves and associated frequencies for a precessing disc around $4 \mathrm{U} 0142+61$ distant $3.6 \mathrm{kpc}$ from the Solar System (Olausen \& Kaspi 2014) are reported in Table 1. In the same Table, similar estimates are reported for a disc possibly precessing around Crab located at $2 \mathrm{kpc}$ from Earth (Manchester et al. 2005 ) or a similar young pulsar, for which different masses are considered. The inner and outer radii of the disc are set equal to $8 \times 10^{7}$ $\mathrm{m}$ and $1.1 \times 10^{8} \mathrm{~m}$, respectively, as lower limits. Wobble angles of 10 and 30 degrees are considered in Tables 1 and 2 . The $\mathrm{S} / \mathrm{N}$ ratio reported in the same tables appears $>1$ up to maximum distances of tens of $\mathrm{kpc}$ for disc masses larger than $10^{28} \mathrm{~kg}$ and wobble angles of the order of tens of degrees. The maximum sensitivity of LISA is $2.5 \times 10^{-22}$ at $3 \mathrm{mHz}$. It is found that the disc around $4 \mathrm{U} 0142+61$ cannot be detected by the LISA interferometer. As it can be also noted in Figure 2. ALIA is supposed to be characterized by a slightly better minimum sensitivity of $5.6 \times 10^{-23}$ but in the frequency band $10^{-2}$ $10^{-1} \mathrm{~Hz}$. DECIGO and BBO are designed for maximum sensitivities

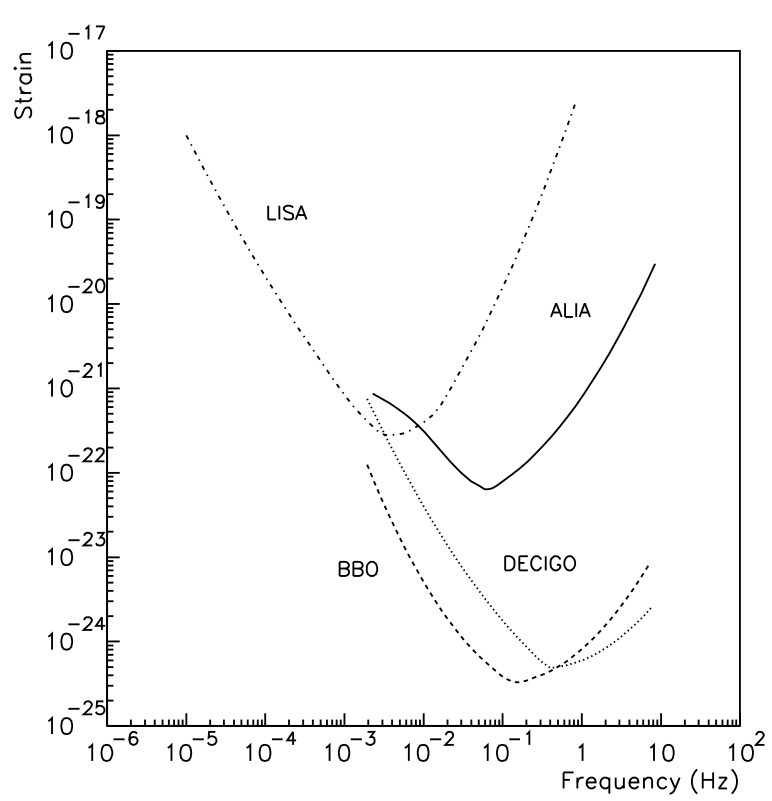

Figure 2. Comparison among sensitivities of the future interferometers for gravitational wave detection in space.

of $5.7 \times 10^{-25}$ and $3.3 \times 10^{-25}$, respectively, between $10^{-2} \mathrm{~Hz}$ and 1 Hz. In the case of gravitational wave emission by precessing discs with masses up to 3.5 orders of magnitude larger than that formed around $4 \mathrm{U} 0142+61$, BBO or DECIGO would be sensitive enough for the detection in case of wobble angles of a few tens of degrees. Moreover, the integration of continuous gravitational wave signals would improve the $\mathrm{S} / \mathrm{N}$ ratio with the square root of time allowing us to resolve these sources with respect to intermediate mass black hole merging in the same frequency range.

Even if the component of the radiation reaction torque perpendicular to the angular momentum is taken into account, the wobble angle change can be expressed as a function of $\theta$ as follows:

$\dot{\theta}=-\frac{1}{\tau_{\theta}} \theta$,

where:

$\frac{1}{\tau_{\theta}}=\frac{2 G}{5 c^{5}} \frac{(\Delta I)^{2}}{I_{1}} \omega^{4}$.

The disc precession lifetime in all considered cases results to be larger than one million year. As a result, the actual capability of the space interferometers to detect precessing discs would depend on the space mission elapsed time.

\section{HIGH-ENERGY POSITRON AND ELECTRON PROPAGATION IN THE REGION OF DISC FORMATION}

In order to estimate the effects of a disc (precessing or not) formed around a young pulsar such as Crab in limiting the propagation of $\mathrm{TeV}$ electrons and positrons, pulsar and disc blackbody radiations are considered and compared. 
Table 1. Gravitational wave amplitude generated by precessing discs and detection distances for $\theta=10^{\circ}$. The signal-to-noise ratio (S/N) is inferred from the LISA (below $10^{-3} \mathrm{~Hz}$ ) and BBO (above $10^{-3} \mathrm{~Hz}$ ) sensitivities reported in Figure 2. Maximum distances for precessing disc detection, when feasible, are set for $\mathrm{S} / \mathrm{N}=1$. The detection probability would increase with the square root of the observational time.

\begin{tabular}{llllll}
\hline & $\begin{array}{l}\text { Disc mass } \\
(\mathrm{kg})\end{array}$ & $\begin{array}{l}\omega_{1}\left(\omega_{2}\right) \\
(\mathrm{Hz})\end{array}$ & $\mathrm{h}_{\omega_{1}}\left(\mathrm{~h}_{\omega_{2}}\right)$ & $\mathrm{S} / \mathrm{N}_{\omega_{1}}\left(\mathrm{~S} / \mathrm{N}_{\omega_{2}}\right)$ & $\begin{array}{l}\text { Distance } \\
(\mathrm{kpc})\end{array}$ \\
\hline 4U 0142+61 & $5.97 \times 10^{25}$ & $3.1 \times 10^{-4}\left(6.2 \times 10^{-4}\right)$ & $1.1 \times 10^{-26}\left(4.5 \times 10^{-26}\right)$ & $<<1(<<1)$ & $0.05(0.05)$ \\
\hline Crab & $5.97 \times 10^{25}$ & $3.9 \times 10^{-2}\left(7.8 \times 10^{-2}\right)$ & $6.7 \times 10^{-26}\left(2.7 \times 10^{-25}\right)$ & $<<1(<1)$ & $0.05(0.05)$ \\
& $10^{27}$ & $“$ & $1.1 \times 10^{-24}\left(4.5 \times 10^{-25}\right)$ & $<1(1)$ & $0.05(0.5)$ \\
& $10^{28}$ & $\cdots$ & $9.3 \times 10^{-25}\left(4.8 \times 10^{-25}\right)$ & $1(1)$ & $0.6(4.65)$ \\
& $10^{29}$ & “ & $8.7 \times 10^{-25}\left(4.5 \times 10^{-25}\right)$ & $1(1)$ & $6.8(50.0)$ \\
\hline
\end{tabular}

Table 2. Same as Table 2 for $\theta=30^{\circ}$. For masses of $10^{29} \mathrm{~kg}$, in principle, disc precession detection may extend to the whole Galaxy (WG).

\begin{tabular}{llllll}
\hline & $\begin{array}{l}\text { Disc mass } \\
(\mathrm{kg})\end{array}$ & $\begin{array}{l}\omega_{1}\left(\omega_{2}\right) \\
(\mathrm{Hz})\end{array}$ & $\mathrm{h}_{\omega_{1}}\left(\mathrm{~h}_{\omega_{2}}\right)$ & $\mathrm{S} / \mathrm{N}_{\omega_{1}}\left(\mathrm{~S} / \mathrm{N}_{\omega_{2}}\right)$ & $\begin{array}{l}\text { Distance } \\
(\mathrm{kpc})\end{array}$ \\
\hline 4U 0142+61 & $5.97 \times 10^{25}$ & $3.5 \times 10^{-4}\left(7.0 \times 10^{-4}\right)$ & $1.9 \times 10^{-25}\left(4.8 \times 10^{-25}\right)$ & $<<1(<<1)$ & $0.05(0.05)$ \\
\hline Crab & $5.97 \times 10^{25}$ & $4.4 \times 10^{-2}\left(8.8 \times 10^{-2}\right)$ & $7.2 \times 10^{-25}\left(4.8 \times 10^{-25}\right)$ & $1(1)$ & $0.05(0.3)$ \\
& $10^{27}$ & $\cdots$ & $8.0 \times 10^{-25}\left(4.2 \times 10^{-25}\right)$ & $1(1)$ & $0.75(5.7)$ \\
& $10^{28}$ & $\cdots$ & $8.1 \times 10^{-25}\left(4.2 \times 10^{-25}\right)$ & $1(1)$ & $7.5(57.1)$ \\
& $10^{29}$ & $\cdots$ & $7.1 \times 10^{-25}\left(4.1 \times 10^{-25}\right)$ & $1(1)$ & $84.5(\mathrm{WG})$ \\
\hline
\end{tabular}

The blackbody photon density near a disc is estimated as follows:

$\frac{N}{V}=8 \pi\left(\frac{k T}{h c}\right)^{3} \times 2.405$

for the temperatures of $920 \mathrm{~K}$ and $1800 \mathrm{~K}$ and $\mathrm{V}=1 \mathrm{~cm}^{3}$, N results to be $1.57 \times 10^{10}$ photons $\mathrm{cm}^{-3}$ and $1.18 \times 10^{11}$ photons $\mathrm{cm}^{-3}$, respectively. The photon average energy associated with the disc temperature of $920 \mathrm{~K}$ and $1800 \mathrm{~K}$ is $0.21 \mathrm{eV}$ and $0.43 \mathrm{eV}$ in the near-infrared range on the basis of equation 2 . In the following we focus in particular on disc temperature of $1800 \mathrm{~K}$ to study the maximum effect of a disc in limiting $\mathrm{TeV}$ energy electron and positron propagation.

The pulsar blackbody radiation intensity estimated in the disc region according to the Stefan-Boltzmann law would be $2.7 \times 10^{7} \mathrm{~W}$ $\mathrm{m}^{-2}$. However, due to the higher energy of the radiation with respect to that of the disc, the photon density from the pulsar results to be $1.15 \times 10^{9}$ photons $\mathrm{cm}^{-3}$, two orders of magnitude smaller than that near the disc. The infrared radiation density from the pulsar would be approximately one order of magnitude smaller than that of the disc (Penny 1982).

The Klein-Nishina IC total cross section of $\mathrm{TeV}$ electrons and positrons scattering low-energy photons to $\mathrm{TeV}$ energies must be considered since the photon energy, $\mathrm{h} v_{O}$, in the rest frame of electrons and positrons is $\mathrm{h} v_{o} /\left(\mathrm{m}_{e} \mathrm{c}^{2}\right)>1$ with $\mathrm{m}_{e} \mathrm{c}^{2}$ being the electron mass. It is worthwhile to recall that the maximum photon energy in the electron rest frame is $\mathrm{h} v_{O}=\mathrm{h} \gamma v_{L}(1+\beta)$ corresponding to a maximum frequency of $2 \gamma v_{L}$ where $v_{L}$ is the frequency of the photon in the laboratory frame, $\beta=\mathrm{v} / \mathrm{c}$ represents the electron speed with respect to the light speed and $\gamma=1 / \sqrt{1-\beta^{2}}$. Since $\gamma=5 \times 10^{6}$ for the photons observed by MAGIC, as a case study, the scattering of infrared radiation leads to $\mathrm{h} v_{o} /\left(\mathrm{m}_{e} \mathrm{c}^{2}\right) \simeq 8>1$.

The total Klein-Nishina cross section is given by:

$$
\begin{aligned}
\sigma_{K N}= & \sigma_{T} \frac{3}{4}\left[\frac{1+x}{x^{3}}\left(\frac{2 x(1+x)}{1+2 x}-\ln (1+2 x)\right)+\frac{1}{2 x} \ln (1+2 x)+\right. \\
& \left.-\frac{1+3 x}{(1+2 x)^{2}}\right]
\end{aligned}
$$

where $x=\mathrm{h} v_{o} /\left(\mathrm{m}_{e} \mathrm{c}^{2}\right)$. Since $\mathrm{h} v_{o} /\left(\mathrm{m}_{e} \mathrm{c}^{2}\right)>1$ the above equation can be reduced as follows:

$\sigma_{K N}=\sigma_{T} \frac{3}{8} \frac{1}{x}\left(\ln 2 x+\frac{1}{2}\right)$

The total cross section and associated collision length would be $9.9 \times 10^{-30} \mathrm{~m}^{2}$ and $8.6 \times 10^{11} \mathrm{~m}$, respectively. Only discs of AU dimensions similar to that of RX J0806.4-4123 with temperatures of at least hundreds of degrees would contribute to $\mathrm{TeV} \mathrm{e}^{+}$and $\mathrm{e}^{-}$IC scattering of infrared photons to $\mathrm{TeV}$ energies. No claim can be made of a large disc surrounding Crab due to continuous electron synchrotron emission observed to dominate the infrared emission in the range 3.6-4.5 $\mu \mathrm{m}$ while $24-70 \mu \mathrm{m}$ emission is associated with less than one solar mass dust in the Nebula (Temim et al. 2006; Lyne et al. 2014).

\section{CONCLUSIONS}

In the next few years, the James Webb telescope will open a new window on the infrared Universe, by shedding light on supernova fallback disc formation around pulsars. Simulations indicate that fallback disc formation may likely occur in a large sample of pulsars and magnetars. A modulated hard X-ray emission from the AXP 4U $0142+61$ observed by the NuSTAR experiment is consistent with a precessing Keplerian disc formed around the magnetar.

Gravitational waves generated by the precession of a disc similar to that observed around $4 \mathrm{U} 0142+61$, would be characterized by frequencies belonging to the LISA detection band but also by amplitudes lying below the sensitivity of the interferometer, while gravitational waves generated by precessing discs formed around young pulsars appear detectable with future generation BBO and DECIGO space interferometers up to tens of $\mathrm{kpc}$ distance and beyond in case of disc large masses, hundreds of kelvin temperature and wobble angle of tens of degrees. It was also shown that the infrared radiation emission from discs of $\mathrm{AU}$ dimensions may contribute to IC scattering affecting high-energy electron and positron propagation in the pulsar near environment. 


\section{ACKNOWLEDGEMENTS}

This research work was funded by the Department of Pure and Applied Sciences of the University of Urbino Carlo Bo. The author thanks M. Fabi of the University of Urbino Carlo Bo for technical support and Dr. F. Sabbatini for reading the manuscript.

\section{DATA AVAILABILITY}

No new data were generated or analysed in support of this research.

\section{REFERENCES}

Aguilar M., et al., 2019, Phys. Rev. Lett., 122, 041102

Aguilera D. N., Pons J. A., Miralles J. A., 2008, The Astrophysical Journal, 673, L167

Alpar M. A., 2001, The Astrophysical Journal, 554, 1245

Amaro-Seoane P., et al., 2017, arXiv e-prints, p. arXiv:1702.00786

Ansoldi S., et al., 2016, A\&A, 585, A133

Armano M., et al., 2018, Physical Review Letters, 120, 061101

Attallah R., 2016, J. Cosmology Astropart. Phys., 2016, 025

Benli O., Ertan Ü., 2017, Monthly Notices of the Royal Astronomical Society, 471,2553

Benli O., Ertan Ü., 2018, Monthly Notices of the Royal Astronomical Society, 478,4890

Benli O., Çalişkan Ş., Ertan Ü., 2015, Monthly Notices of the Royal Astronomical Society, 447, 2282

Chatterjee P., Hernquist L., Narayan R., 2000, The Astrophysical Journal, 534,373

Currie T., Hansen B., 2007, The Astrophysical Journal, 666, 1232

Cutler C., Harms J., 2006, Phys. Rev. D, 73, 042001

Diesing R., Caprioli D., 2020, Phys. Rev. D, 101, 103030

Durant M., van Kerkwijk M. H., 2006, The Astrophysical Journal, 652, 576

Erdeve I., Kalemci E., Alpar M. A., 2009, The Astrophysical Journal, 696, 1792

Ertan Ü., Erkut M. H., Ekşi K. Y., Alpar M. A., 2007, The Astrophysical Journal, 657, 441

Ertan Ü., Ekşi K. Y., Erkut M. H., Alpar M. A., 2009, The Astrophysical Journal, 702, 1309

Ertan Ü., Çalişkan Ş., Benli O., Alpar M. A., 2014, Monthly Notices of the Royal Astronomical Society, 444, 1559

Feng J., Zhang H.-H., 2018, The Astrophysical Journal, 858, 116

Gardner J. P., et al., 2006, Space Sci. Rev., 123, 485

Gençali A. A., Ertan Ü., 2020, Monthly Notices of the Royal Astronomical Society, 500,3281

Grimani C., 2004, A\&A, 418, 649

Grimani C., 2007, A\&A, 474, 339

Grimani C., 2013, Advances in Space Research, 51, 322

Grimani C., 2016, Monthly Notices of the Royal Astronomical Society, 460, 2186

Güver T., Özel F., Göğüş E., 2008, The Astrophysical Journal, 675, 1499

Harding A. K., Ramaty R., 1987, in International Cosmic Ray Conference. p. 92

Hawley J. F., Krolik J. H., 2018, The Astrophysical Journal, 866, 5

Huang Z.-Q., Liu R.-Y., Joshi J. C., Wang X.-Y., 2020, The Astrophysical Journal, 895, 53

Jiang Z.-B., Li X.-D., 2005, ] 10.1088/1009-9271/5/5/006, 5, 487

Kaplan D. L., Chakrabarty D., Wang Z., Wachter S., 2009, The Astrophysical Journal, 700, 149

Kawamura S., et al., 2020, arXiv e-prints, p. arXiv:2006.13545

Lee H. K., Lee C. H., Kim H. S., 2004, Journal of the Korean Physical Society, 45,564

Li X.-D., 2006, Astrophys. J., 646, L139

Lu Y., Cheng K. S., 2002, Chinese Journal of Astronomy and Astrophysics, 2,161
Lyne A. G., Jordan C. A., Graham-Smith F., Espinoza C. M., Stappers B. W., Weltevrede P., 2014, Monthly Notices of the Royal Astronomical Society, 446, 857

Makishima K., Murakami H., Enoto T., Nakazawa K., 2018, Publications of the Astronomical Society of Japan, 71

Manchester R. N., Hobbs G. B., Teoh A., Hobbs M., 2005, The Astronomical Journal, 129, 1993

Manconi S., Di Mauro M., Donato F., 2020, Phys. Rev. D, 102, 023015

Menou K., Perna R., Hernquist L., 2001, The Astrophysical Journal, 554, L63

Ni W.-T., 2016, International Journal of Modern Physics D, 25, 1603001

Olausen S. A., Kaspi V. M., 2014, The Astrophysical Journal Supplement Series, 212, 6

Özcan Ş., Gençali A. A., Ertan Ü., 2020, Monthly Notices of the Royal Astronomical Society, 498, 674

Penny A. J., 1982, Monthly Notices of the Royal Astronomical Society, 198, 773

Perna R., Hernquist L., Narayan R., 2000, The Astrophysical Journal, 541, 344

Posselt B., Pavlov G. G., Ertan Ü., Çalişkan Ş., Luhman K. L., Williams C. C., 2018, The Astrophysical Journal, 865, 1

Qiao Xue, Y. Q. Xu, R. X. Wang, H. G. Xiao, B. W. 2003, A\&A, 407, L25

Shakura N. I., Sunyaev R. A., 1973, A\&A, 500, 33

Steiner A. W., Heinke C. O., Bogdanov S., Li C. K., Ho W. C. G., Bahramian A., Han S., 2018, Monthly Notices of the Royal Astronomical Society, 476,421

Temim T., et al., 2006, The Astronomical Journal, 132, 1610

Tennant A. F., et al., 2001, The Astrophysical Journal, 554, L173

Tong H., Song L. M., Xu R. X., 2011, ApJ, 738, 31

Tong H., Wang W., Wang H.-G., 2020, Research in Astronomy and Astrophysics, 20, 142

Wang Z., Chakrabarty D., Kaplan D. L., 2006, Nature, 440, 772

Wolszczan A., 2008, Physica Scripta Volume T, 130, 014005

Zhang L., Cheng K. S., 2001, A\&A, 368, 1063

This paper has been typeset from a $\mathrm{TE}_{\mathrm{E}} \mathrm{L} / \mathrm{AT} \mathrm{E} \mathrm{X}$ file prepared by the author. 